\title{
Bulk Metallic Glass Based Tool-making Process Chain for Micro- and Nano- Replication
}

\author{
Pierre C. Vella ${ }^{1,3}$, Stefan S. Dimov ${ }^{1}$, Alexander Kolew², Ekaterin Minev², Krastimir Popov², \\ Franck Lacan ${ }^{2}$, Christian Griffiths ${ }^{2}$, Hassan Hirshy ${ }^{2}$ and Steffen Scholz ${ }^{2}$ \\ ${ }^{1}$ School of Mechanical Engineering, University of Birmingham, Edgbaston, Birmingham, B15 2TT, UK \\ ${ }^{2}$ Manufacturing Engineering Centre, Cardiff School of Engineering, Cardiff University, Cardiff CF24 3AA, UK \\ ${ }^{3}$ Department of Industrial and Manufacturing Engineering, University of Malta, Msida MSD2080, Malta
}

\begin{abstract}
Existing and emerging micro-engineered products tend to integrate a multitude of functionalities into single enclosures/packages. Such functions generally require different length scale features. In practice, devices having complex topographies, which incorporate different length scale features cannot be produced by employing a single fabrication technology but by innovatively, integrating several different complementary manufacturing techniques in the form of a process chain. In order to design novel process chains that enable such function and length scale integration into miniaturised devices, it is required to utilise materials that are compatible with the various component manufacturing processes in such chains. At the same time, these materials should be able to satisfy the functional requirements of the produced devices. One family of materials, which can potentially fulfil these criteria, is bulk metallic glasses (BMGs). In particular, the absence of grain boundaries in BMGs makes them mechanically and chemically homogeneous for processing at all length scales down to a few nanometres. In this context, this research presents an experimental study to validate a novel process chain. It utilizes three complementary technologies for producing a Zr-based BMG replication master for a microfluidic device that incorporates micro and nano scale features. Then, to validate the viability of the fabricated BMG masters, they are utilized for serial replication of the microfluidic device by employing micro-injection moulding.
\end{abstract}

Keywords: Micromilling, Hot Embossing, Focused Ion Beam Milling, Bulk Metallic Glasses, Process Chains, Function and Length Scale Integration.

\section{Introduction}

Existing high-tech and new emerging products clearly show that there is a trend for integrating multiple functions in as small as possible enclosures/packages. Such systems generally include functions that require different length scale features, for instance nanoelectronics, various microsensors, micro and/or nano actuators or microfluidic devices encapsulated in a single container [1]. However, the range of available micro and nano manufacturing technologies that underpin the development of such products are limited in their capabilities for producing $3 \mathrm{D}$ micro and nano structures in a wide range of materials [2]. Therefore, in practice, devices having complex topographies, which incorporate different length scale features cannot be produced by employing a single fabrication technology, but by innovatively integrating several different complementary manufacturing techniques in the form of a process chain. Thus, it is not surprising that the design and validation of such process chains has attracted the attention of researchers and some successful implementations have been reported to satisfy the specific functional and technical requirements of emerging multi-material products $[3,4,5]$. However further research is necessary to address many challenges that arise in achieving a length-scale integration and 3D patterning simultaneously by applying a given process chain. Especially, in developing such process chains it is necessary to address various specific process integration issues and at the same time to satisfy the functional requirements of the produced device/replication master. The requirement to utilize materials that are compatible with all the manufacturing processes integrated in a chain is such an important consideration. Thus, when designing a process chain and selecting an appropriate material to realize the requirements of the product or replication master, the processing constraints of the individual micro and nano manufacturing technologies that are integrated in the chain also have to be taken into account. This means that the selected material should have a microstructure with a favourable response to structuring both at meso/macro and micro/nano scales. In particular, to undergo micro or nano structuring successfully, such a material should have specific characteristics, such as being homogeneous and inclusion free at the considered processing scales. Thus, the material properties of a workpiece are a critical factor affecting the machining results and the predictability in micro and nano manufacturing. Due to their homogeneous microstructure, the absence of any lattice defects and of grain boundaries [6], amorphous metallic alloys are considered promising materials for

Proceedings of the 9th International Conference on Multi-Material Micro Manufacture Edited by Humbert Noll, Nadja Adamovic and Stefan Dimov Copyright (c) 2012 4M 2012 Organisers :: Published by Research Publishing 
micro and nano- structured replication masters $[7,8]$. In particular, the absence of grain boundaries in metallic glasses (MG) makes them mechanically and chemically homogeneous for processing at all length scales down to a few nanometres. As a result, they are one of the preferred materials for micro- and nano structuring. [9]. In this context, this paper presents an experimental study to validate a novel process chain. It utilizes three complementary technologies for producing a bulk metallic glass (BMG) based replication master for a microfluidic device that incorporates micro and nano scale features. Then, to validate the viability of the fabricated BMG masters, they were utilized for serial replication of the microfluidic device by employing micro-injection moudling.

The paper is organized as follows. Section 2 presents the design of the proposed master making process chain. Section 3 outlines the experimental set-up utilised in this study for producing the masters and then for validating their replication capabilities. Finally, the obtained results are discussed and conclusions are made in Sections $5 \& 6$, respectively.

\section{Process Chain Design}

The proposed process chain is shown in Fig. 1. A brief overview of this process chain is given below. To demonstrate its feasibility, the process chain is utilsed to fabricate microfluidic devices incorporating micro- and nano-scale features. The first step of the proposed process chain is the manufacture of an aluminium tool by micro milling that has the same design as the final product. It is important to note that the master material was selected on the basis of its machinability and the requirement to dissolve it once the hot embossing process is complete. In the second step, the aluminium master was integrated into a hot embossing (HE) machine to transfer the microfliudic device micro-scale features onto the $\mathrm{Zr}$ based BMG. To benefit from the outstanding mechanical and physical properties of this material and thus realise successfully the proposed master making process chain, it is of prime importance to maintain its non-crystalline morphology by optimising the HE process variables. The third step employs Focused Ion Beam (FIB) milling to produce sub-micron structures on top of a pre-existing micro features. This technology can be applied for patterning accurately and cost effectively only relatively small areas, e.g. up to $100 \times 100 \mu \mathrm{m}$ without stitching, due to its low material removal rates and thus it is not suitable for structuring bigger surfaces. Hence, to make the best use of the FIB milling process, it was applied only for patterning a relatively small area with submicron and nano-structures. Finally, the micro- and nanostructured BMG insert was integrated into a injection moudling machine and small batches of thermoplastic polymer based microfluidic devices were produced.

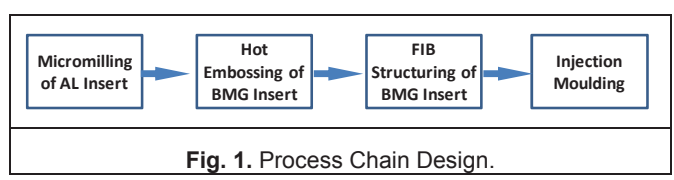

\section{Experimental Setup}

\subsection{Test Structure Design}

The test structure was selected to demonstrate the feasibility of producing sub micron and nano scale structures directly onto the micro-scale features of the
BMG tool, and thus to transfer in a single step both micro and nano scale features onto the surfaces of polymer replicas. A schematic view of the micro channels and reservoirs of the simple micro-mixer used in this study is shown in Fig. 2. Three 2D grating with sub micron and nano scale patterns are located into the large reservoir of the microfluidic device as indicated in Fig. 2. Each pattern consists of $20 \times 20$ fields of black or white squares and all black fields are pockets of specific depths. The dimensions for these three grating structures are shown in Fig. 3.

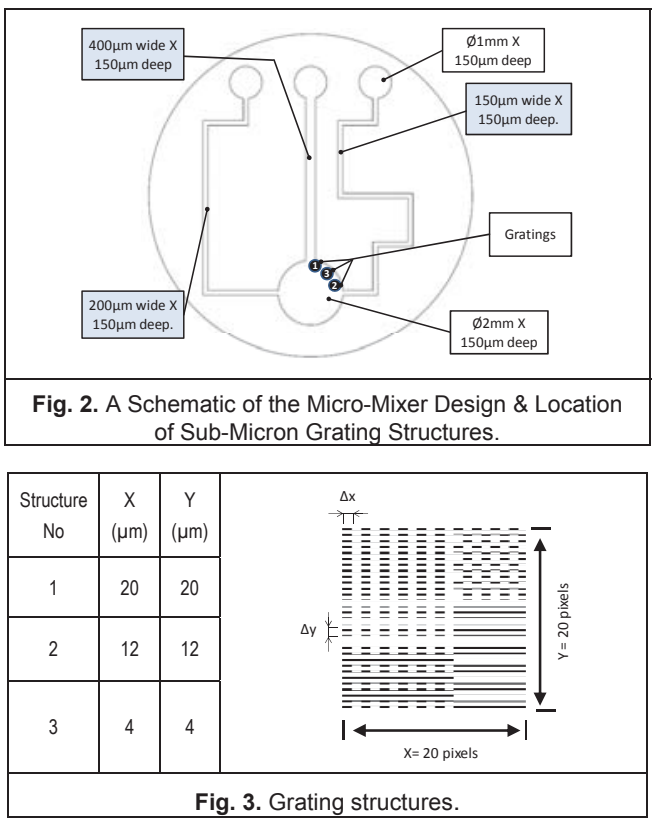

\subsection{Micromilling of Aluminium Master}

Micromilling is still the most cost effective technology for producing 3D complex micro structures [10]. Thus, this technology was employed in the proposed process chain to machine the micro-mixer features onto the aluminium workpiece. A standard grade of aluminium was used, in particular AL5083. This aluminium alloy was selected because of its machinability [11] and the requirement to dissolve it after completing the hot embossing stage. The machining of the aluminium masters was carried out on a KERN HSPC 2216 micro-machining centre. The factors affecting the performance of micro milling operations investigated in a previous study [12] were taken into account in selecting the machining parameters in Table 1 for producing the Al master. The channels and reservoirs were machined to their full depth of $150 \mu \mathrm{m}$ by applying reciprocating plunge-cut cycles. A $150 \mu \mathrm{m}$ diameter end-mill was used to mill the channels and the reservoirs.

Table 1. Micro milling Process Parameters.

\begin{tabular}{|l|l|}
\hline Feed Rate $(\mathrm{mm} / \mathrm{min})$ & 250 \\
\hline Spindle speed $(\mathrm{RPM})$ & 40,000 \\
\hline Depth of Cut $(\mu \mathrm{m})$ & 2 \\
\hline Step Over $(\mu \mathrm{m})$ & 50 \\
\hline
\end{tabular}




\subsection{Material Selection}

The particular BMG used in this study is Vitreloy $1 \mathrm{~b}$ (Vit1b). The mechanical properties of this alloy are extremely high such as its tensile yield strength of $1.9 \mathrm{GPa}$ and a hardness of $540 \mathrm{Hv}$. Therefore, it is a material of interest to produce high wear resistant mould inserts for micro injection moulding. Furthermore, it is expected that a high surface integrity can be achieved when replicating and machining micro and nano-structures into this material. This is due to the fact that no crystalline defects, such as dislocation pile-ups, point defect agglomerates and grain boundaries are present in the material and thus, this prevents the formation of micro-cracks and pores during processing.

\subsection{Hot Embossing of BMG Master}

Micro Hot Embossing, a specific thermoplastic forming process suitable for producing microscale, high aspect ratio, patterned features on metallic glass plates[13] was employed in this research. In particular, a hot embossing machine developed at the Institute of Microstructure Technology (KIT) and based on a Zwick universal testing machine with short cycle times was used to produce the $\mathrm{Zr}$-based BMG master. The forming is performed by a displacement and force controlled embossing step, which is controlled by a high precision load cell. To ensure homogenous temperature and pressure distribution during the replication process, the force and temperature were kept constant for a predefined time that was selected taking into account the structure design, and thermal conductivity and flow ranges [14] of the BMG. A circular flat workpiece of Vitreloy $1 \mathrm{~b}$ BMG having a diameter of $15 \mathrm{~mm}$ was placed together with the micromixer patterned aluminium tool between the parallel heated compression platens. The BMG was then heated to a temperature above the transition temperature, and then a defined pressure was applied over a set amount of time. After that, the load was removed and the Al masterBMG assembly was cooled. It is important to stress that after a certain amount of time at a given temperature above the glass transition temperature, the Zr-based BMG will start crystallising, which is undesirable because it leads to degrading substantially the properties of the formed part. Therefore, it is imperative to consider this time constraint when selecting appropriate temperaturetime processing parameters for the micro hot embossing process.

The mechanical behaviour of BMGs is highly temperature dependent above their glass transition temperature, in particular the 'viscosity' of the material decreases dramatically as the temperature increases, and, as such, selecting a temperature as high as possible without risking crystallization is the most important consideration in selecting the processing window [13]. Due to the high cost of the material, only five hot embossing trials were conducted to select the process settings and generate the BMG inserts. Also, in conducting these trials the results from another study [15] were taken into account, which suggested for the Vit $1 \mathrm{~b}$, that at a processing temperature of $460^{\circ} \mathrm{C}$, crystallisation becomes detectable after $255 \mathrm{~s}$. Within this context, for the trials in this study, a maximum temperature of $450^{\circ} \mathrm{C}$ and an overall process time including heating, forming and cooling below $200 \mathrm{~s}$ were chosen for embossing the Vitreloy-1b work piece, since the risk of crystallisation and the subsequent deterioration of mechanical properties of the BMG under these process conditions was expected to be minimal.
Subsequently, on the basis of these trials, it was found that for the geometry under consideration, a temperature of $450^{\circ} \mathrm{C}$, and a force of $5000 \mathrm{~N}$ would result in a completely filled mould after $190 \mathrm{~s}$. Following the embossing stage, the BMG was separated from the aluminium master by dissolving it in a heated $\mathrm{NaOH}$ bath. Finally, any flashes occurring as a result of the hot embossing process may be trimmed at this point by wire EDM and thus to produce the final insert with the required overall size for integrating it into a injection moulding tool.

\subsection{FIB Structuring}

The next stage in the process chain was the FIB machining of the sub-micron and nano scale features of the three grating structures (See Figs. 2 and 3 ) over the embossed micro-fluidic reservoir. The three structures were machined onto the BMG workpiece using a Carl Zeiss XB 1540 FIB/SEM system that combines a gallium ion beam with an electron beam column. The FIB milling process can be controlled by utilising a built-in software or an external nanolithography system [16]. Given that the three gratings represent a 2D image composed of black and white fields/pixels, the bitmap file of this pattern was used directly by the built-in software of the FIB system to control the milling operation. The depth and accuracy of the structures fabricated by FIB milling are determined by the processing parameters used. In this research, the process was set up and tuned by conducting the necessary machining trials to find out quickly a suitable processing window, whilst also achieving the best trade-offs between machining time and resulting surface quality. The processing parameters used in this experimental study are provided in Table 2. The alignment of the gratings over the micro mixer reservoir was performed manually using the FIB imaging mode of the system.

Table 2. FIB Milling Parameters.

\begin{tabular}{|l|c|c|c|}
\hline Structure No. & $\mathbf{1}$ & $\mathbf{2}$ & $\mathbf{3}$ \\
\hline Probe current & $2 \mathrm{nA}$ & $200 \mathrm{pA}$ & $10 \mathrm{pA}$ \\
\hline Accelerating voltage & $30 \mathrm{kV}$ & $30 \mathrm{kV}$ & $30 \mathrm{kV}$ \\
\hline Exposure time duration & $489 \mathrm{~s}$ & $963 \mathrm{~s}$ & $1070 \mathrm{~s}$ \\
\hline
\end{tabular}

\subsection{Micro Injection Moulding}

Micro injection moulding is one of the key technologies for reliable and cost effective manufacturing of polymer micro devices. [5] The machine used to perform the micro injection moulding tests was a Battenfeld Microsystem 50 . First, the BMG insert was integrated into the primary mould. An existing secondary insert holder was modified to accommodate and hold the BMG insert. A packing block was then fitted to the secondary mould insert holder to secure the smaller BMG insert into place. This assembly was fitted into the primary mould as seen in Fig. 4.

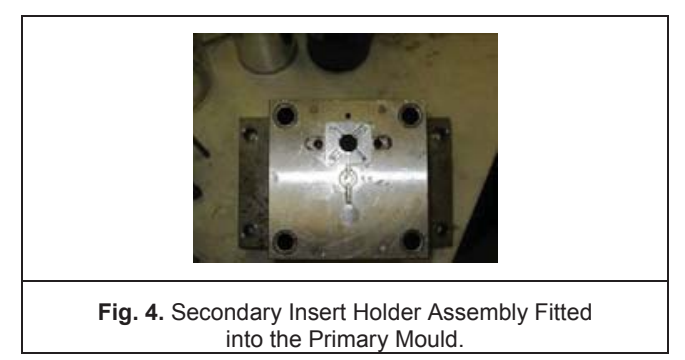




\section{Humbert Noll, Nadja Adamovic and Stefan Dimov (Eds.)}

The overall design included a circular main body with dimensions $\varnothing 11 \mathrm{~mm} \times 2 \mathrm{~mm}$ and four "wing" features to assist demoulding. In addition, a gate and a runner were machined into the secondary mould insert holder to allow the plastic to flow from the injection nozzle into the BMG insert.

Three commonly used materials in injection moulding, namely Polypropylene (PP), Polycarbonate (PC) and Polyamide filled with $20 \%$ Glass fibre (PA+20\%GF) were selected to conduct the replication trials. The first two materials were used to demonstrate the validity of this process chain for replicating satisfactorily both the micro and nano scale structures, whilst the third one was selected to investigate the durability of the BMG inserts by conducing 1000 replication cycles with this highly abrasive polymer. The process settings used for the abovementioned materials were selected based on previous experimental studies $[5,17,18]$ in conjunction with the respective material data sheets, and are provided in Table 3. Before starting the replication trials, the polymers were placed in desiccant drying and dehumidifying cycles to remove any surface or absorbed moisture.

\begin{tabular}{|l|l|l|l|}
\hline \multicolumn{4}{|c|}{ Table 3. The Main Injection Moulding Process settings. } \\
\hline Material: & $\begin{array}{l}\text { PP } \\
\text { (PPH } \\
5042)\end{array}$ & $\begin{array}{l}\text { PC (Lexan } \\
\text { HPS1-1124) }\end{array}$ & $\begin{array}{l}\text { PA+GF20\% } \\
\text { (Grivory) }\end{array}$ \\
\hline $\begin{array}{l}\text { Melt temp -- } \mathrm{T}_{\mathrm{b}} \\
\left({ }^{\circ} \mathrm{C}\right)\end{array}$ & 240 & 290 & 260 \\
\hline $\begin{array}{l}\text { Mould Temp -- } \\
\mathrm{T}_{\mathrm{m}}\left({ }^{\circ} \mathrm{C}\right.\end{array}$ & 90 & 90 & 80 \\
\hline $\begin{array}{l}\text { Holding Pres- } \\
\text { sure -- } \mathrm{P}_{\mathrm{h}}\end{array}$ & ON & ON & ON \\
\hline $\begin{array}{l}\text { Injection Speed } \\
--\mathrm{V}_{\mathrm{i}}(\mathrm{mm} / \mathrm{s})\end{array}$ & 200 & 200 & 400 \\
\hline
\end{tabular}

\section{Results and Discussion}

Some of the main experimental results obtained with this experimental study are discussed in this section. Figures 5,6 and 7 depict images of the aluminium master with the micro-scale features produced by micro milling; the BMG insert with the micro- and nano-scale features formed by HE and FIB milling; and the polymer replicas produced through micro injection moulding, respectively.

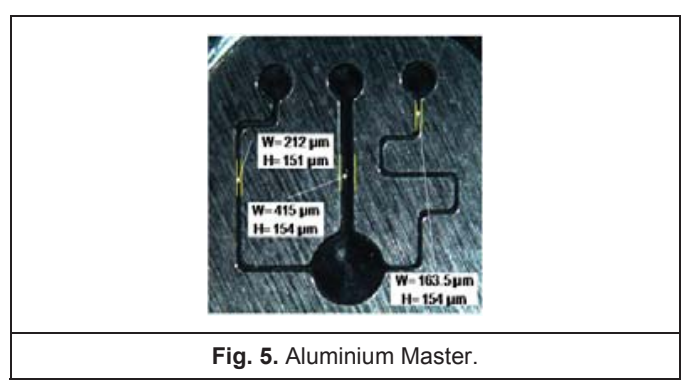

The microscale features' widths and heights of the aluminium master, BMG insert and replicated polymer parts were measured with a Leica DMLM measuring microscope and Mitutoyo QV Accel 404 Measuring machine, respectively. Whilst at the nano-scale, the features' widths and heights were measured using the SEM integrated into the FIB system. The heights were measured by FIB sectioning and then tilting the stage at 54 degrees employing a built-in tilt compensation algorithm for image processing. Studying the images in Figs. 5 and $6 a$, it can be judged that the micro-mixer was hot embossed

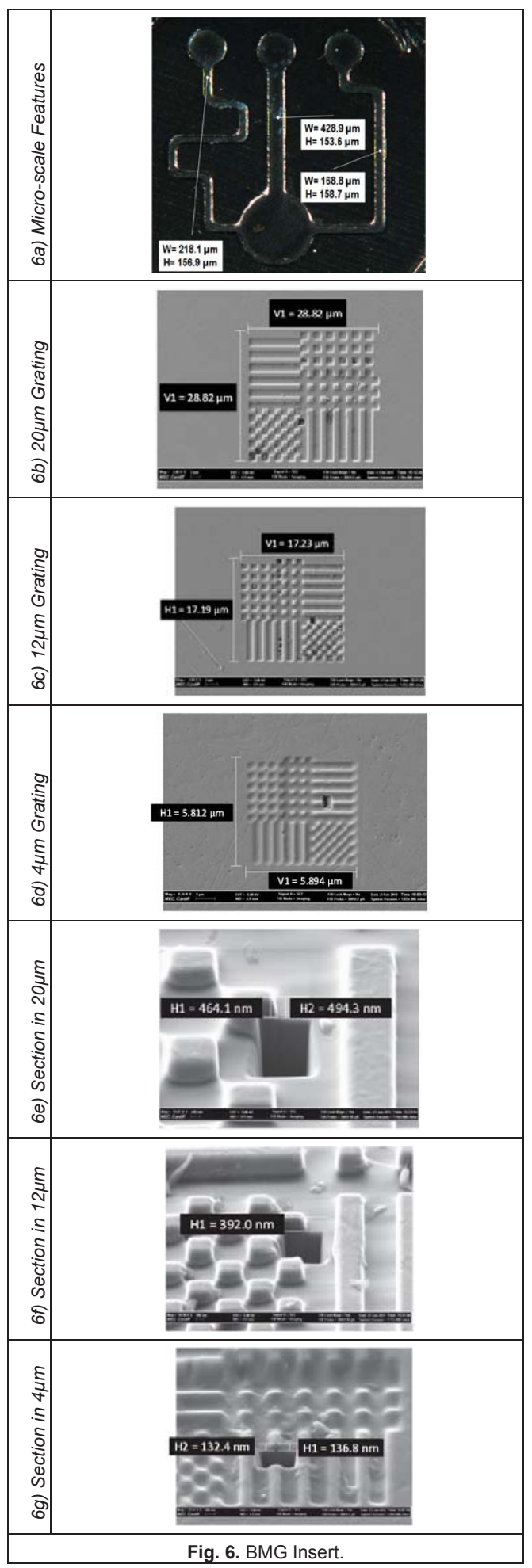




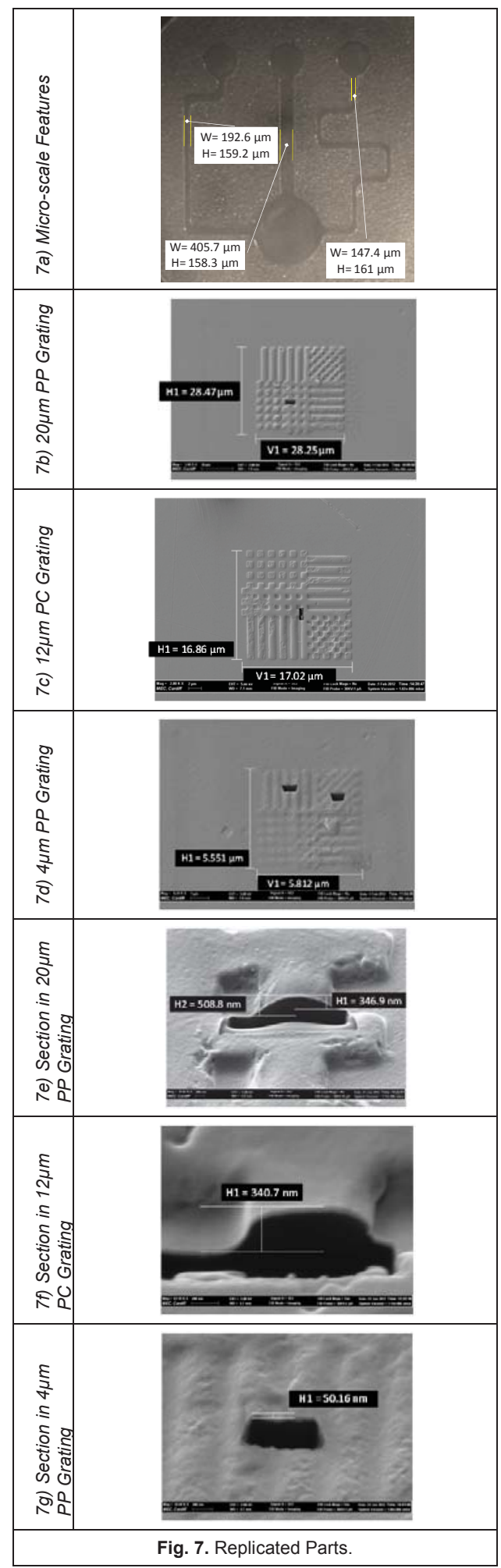

satisfactorily onto the BMG workpiece. Figures $6 b, 6 c$ and $6 \mathrm{~d}$ show respectively that although the three grating patterns were FIB structured satisfactorily on the top surface of the reservoir according to the scaled bitmap image, the measured overall mean dimensions of all the three gratings are larger than the target values of $20 \mu \mathrm{m}$, $12 \mu \mathrm{m}$ and $4 \mu \mathrm{m}$ for structures 1,2 and 3 respectively.

In addition, it can be seen from Figs. $6 e, 6 f$ and $6 \mathrm{~g}$, that the average height of structures 1,2 and 3 was estimated to be $479.2 \pm 45 \mathrm{~nm}, 392 \pm 43 \mathrm{~nm}$ and $134.6 \pm 22$ $\mathrm{nm}$, respectively. The tolerance range for each of these values represents the expanded uncertainty, $\mathrm{U}$, which was determined, following an established procedure [19], out of 6 repeated measurements and includes the uncertainty of the measuring instrument $u(P)$. To account for the worst-case scenario the measurement uncertainty $u(P)$ of the SEM was calculated as $3 \%$ of the measurands' average value. Again, it can be observed that these actual mean heights differ from the targeted heights of $1000 \mathrm{~nm}, 600 \mathrm{~nm}$ and 200nm of these structures. Therefore, further FIB process optimisation is required to improve the dimensional accuracy of the submicron gratings. Utilising the BMG insert, polymer replicas in PP, PC and PA+20\% GF were produced. Fig. 7a shows a micro-mixer replicated in PP. When comparing Figs. $6 a$ and $7 a$, it can be observed that at the microscale, the micro-mixer was replicated satisfactorily. The same can be stated for the sub-micron and nano scale gratings when comparing visually the replication results in Figs. 6b, 6c and 6d with those in Figs. 7b, 7c and 7d. However, when comparing the measurement results in Figs. $6 \mathrm{e}, 6 \mathrm{f}$ and $6 \mathrm{~g}$ with those in Figs. $7 \mathrm{e}, 7 \mathrm{f}$ and $7 \mathrm{~g}$ it can be seen that there are significant differences between the as-measured heights of the replicated parts and the actual heights of the FIB milled structures in the BMG insert. This difference cannot be solely attributed to polymer shrinkage and could suggest that the grating structures were not completely filled by the melt. Nevertheless, these initial results are very encouraging but they also indicate that further work is required to optimise the micro-injection moulding processing parameters and thus to improve the quality of the replicated structures.

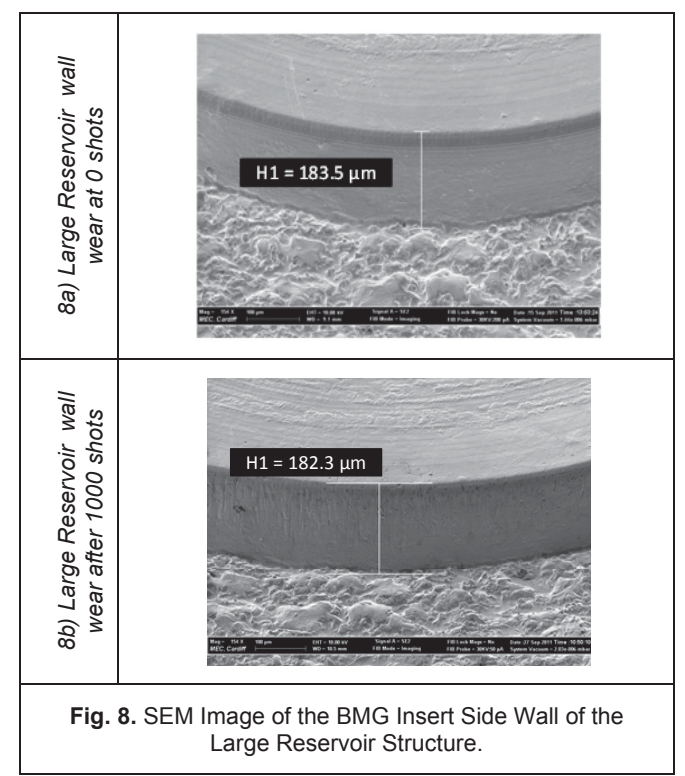




\section{Humbert Noll, Nadja Adamovic and Stefan Dimov (Eds.)}

Finally, Fig. 8 shows SEM-pictures of one specific side wall of the cylindrical test structure (a) before the IM trials, i.e 0 shots, and (b) after 1000 cycles. A smoothening of the initial sharp edge resulting from the milling operation is clearly visible. Especially, the top edge of the reservoir became more and more rounded and grooves from the milling operation are levelled. Also, it can be observed that the upper part of the side wall has experienced a higher wear compared to the lower part. The reason for this is that the upper part is not only strained during the injection moulding shots but also during the whole demoulding process. Therefore, the upper part of the test structure is about twice as long in contact with the abrasive PA $+20 \%$ Glass Fibre than the lower part.

\section{Conclusions}

The paper presented a novel process chain for replicating micro- and nano-scale structured surfaces. A validation study was conducted by producing BMG inserts incorporating micro- and nano scale features and subsequently by replicating them employing micro injection moulding. It has been demonstrated that this indirect tooling making process chain can be a viable fabrication route for BMG inserts. In addition, promising results have been obtained by micro and nano-scale structuring of the BMG workpiece by hot embossing and FIB milling, respectively; and then replicating them to produce parts with nano scale gratings on top of a micro scale reservoir feature of a microfluidic mixer. At the same time, some limitations were observed with respect to the dimensional accuracy of the produced sub-micron and nano-scale features. It was also shown, that after 1000 injection cycles with $\mathrm{PA}+20 \%$ GF, a significant wear of the test structures can be observed. There are changes in the shape of the micro-scale test structures especially on edges caused by the moulding/demoulding cycles. However, despite this, the overall conclusion is that the proposed master making process chain is a promising manufacturing route for high throughput fabrication of polymer parts with complex structures that incorporate different length scale features. However, further research is necessary to develop it as a viable manufacturing route. In particular, it is important to investigate quantitatively the wear of the BMG used in this study, especially by conducting a higher number of injection moulding cycles. Also, the influence of different polymer melts should be investigated. All, this should determine the limitations of the BMG insert when utilised as inserts for micro injection moulding. In addition, it is important to identify optimum processing windows in regards to the quality and accuracy of the replicated nano-scale polymer structures. Such a research can be extended to investigate also the limits of the process chain and the replication technology when it is applied for producing similar but a higher aspect ratio micro and nano scale structures.

\section{Acknowledgements}

The research reported in this paper was carried out within the framework of the EC FP7 projects "Integrating European research infrastructures for the micro-nano fabrication of functional structures and devices out of a knowledge-based multimaterials repertoire" (EUMINAfab)", "Converging technologies for micro systems manufacturing" (COTECH) and "Flexible Compression Injection Moulding Platform for Multi-Scale Surface Structures" (IMPRESS). Also, the authors would like to thank the University of Malta for providing financial support to Mr Pierre Vella's PhD research.

\section{References}

[1] S. Evoy, S. et al , "Dielectrophoretic Assembly and Integration of Nanowire Devices with Functional CMOS Operating Circuitry.," Microelectronic Engineering, vol. 75, 2004, pp. 31-42.

[2] Azcarate, S. et al , "Hybrid Tooling: A Review of Process Chains for Tooling Microfabrication Within 4M," Proc. of the 4M 2006 Conference, 2006, pp. 305-308.

[3] Tosello, G. et al , "High Aspect Ratio Micro Tool Manufacturing for Polymer Replication using $\mu E D M$ of Silicon, Selective Etching and Electroforming," Microsystem Technologies, vol. 14, no. 9-11, 2008, pp. 1757-1764.

[4] Velkova, V. et al "Design and Validation of a Novel Mastermaking Process Chain for Organic and Large Area Electronics on Flexible Substrates," Microelectronic Engineering, vol. 87, no. 11, 2010, pp. 2139-2145.

[5] Scholz, S. et al, " Manufacturing routes for replicating micro and nano surface structures with bio-mimetic applications," CIRP Journal of Manufacturing Science and Technology, Vol. 4 (4), 2011, pp 347-356

[6] Kawasegi, N., et al., "Rapid nanopatterning of a Zr-based metallic glass surface utilizing focused ion beam induced selective etching". Appl. Phys. Lett. 89 , 2006, 143115-3.

[7] Minev R., et al., "Deposition and Focused Ion Beam Milling of Anticorrosive $\mathrm{CrC}$ Coatings on Tool Steel Substrates", The International Journal of Advanced Manufacturing Technology, Vol. 47, Numbers 1-4, 2009, 29-35

[8] Quintana I., et al.,"Investigation of Amorphous and Crystalline Ni Alloys Response to Machining with Micro-second and Pico-second Lasers", Applied Surface Science, 255(13-14), 2009, 6641-6646

[9] Loffler, J.F. et al, Materials Processing Handbook, Groza, J.R. et al (Eds), CRC Press, 2007, pp17-1 - 17-44.

[10] Uriarte et al, "Comparison Between Microfabrication Technologies for Metal Tooling.", Proc Instn Mech Engrs Part C: Journal of Mechanical Engineering Science, Vol. 220, 2006, pp 1665-1676

[11] Popov K, et al , "Micro-milling: Material Microstructure Effects.", Proc. Instn Mech. Engrs Part C, Journal of Mechanical Engineering Science, Vol. 220 (11), 2006, pp 1807-1813

[12] Dimov S, et al , "Micromilling strategies: optimization issues", Proc. of the Instn Mech. Engrs, , Part B, Engineering Manufacture, Vol. 218, 2004, pp.731-736 ]

[13] Henann D.L., "Metallic Glasses: Viable Tool Materials for the Production of Surface Microstructures in Amorphous Polymers by Micro-hot-embossing.", J. Micromech. Microeng., Vol. 19 (11), 2009, pp 1-10

[14] Kolew A. et al, "Hot Embossing of Micro and Sub-micro Structured Inserts for Polymer Replication", Microsystem Technologies, Vol. 17 (4), 2010, pp 609-618.

[15] Waniuk T. et al, "Timescales of Crystallization and Viscous Flow of the Bulk Glass-forming Zr-Ti-Ni-Cu-Be Alloys", Physical Review, 67, 2003, pp 182403-1 - 182403-9.

[16] Lalev, G. et al "Data Preparation for FIB machining of Complex 3D Structures," Proc Instn Mech Engrs Part B: $J$ Engineering Manufacture, vol. 222, no. 1, 2008, pp. 67-76

[17] Griffiths, C.A. et al , "Polymer Inserts Tooling for Prototyping of Micro Fluidic Components in Micro Injection Moulding." Proc. of the 4M 2007 Conference, 2007, pp. 305-308.

[18] Tosello G., Precision Moulding of Polymer Micro Components (PhD Thesis), DTU, 2008, pp 1-250

[19] Kirkup, L., Frenkel, B., 2006, An Introduction to Uncertainty in Measurement, First Edition. Cambridge University Press, Cambridge. 